\title{
Investigating the anatomical relationship between the maxillary molars and the sinus floor in a Chinese population using cone- beam computed tomography
}

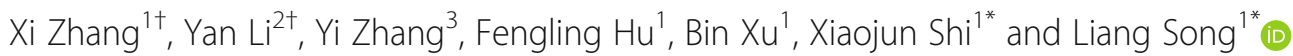

\begin{abstract}
Background: The anatomical relationship between the root apices of maxillary molars and the maxillary sinus floor (MSF) is important for the treatment of dental implantations and endodontic procedures. In this study, the detailed anatomical relationships between the root apices of maxillary molars and the MSF were studied in a Chinese population using CBCT.

Methods: We collected the CBCT data files of patients who visited the stomatology outpatient clinic in Shanghai Fifth People's Hospital, Fudan University from January 1, 2017 to January 1, 2019 and measured the following items: the distance between the molar root apices and the MSF, the thickness of the mucosa and cortical bone of the MSF closest to the root apices, and the angle between the buccal and palatal roots.

Results: The shortest distances between the root apices and the MSF were $1.57 \pm 3.33 \mathrm{~mm}$ (the mesiobuccal root of the left second molar) and $1.61 \pm 3.37 \mathrm{~mm}$ (the mesiobuccal root of the right second molar). Apical protrusion over the inferior wall of the sinus most often occurred in the mesiobuccal root of left second molar (frequency, 20.5\%). The mucosa of the MSF was thinnest at the distobuccal root of the right second molar $(1.52 \pm 0.85 \mathrm{~mm})$, the cortical bone of the MSF was thinnest at the mesiobuccal root of the right second molar $(0.46 \pm 0.28 \mathrm{~mm})$, and the angle between the buccal and palatal roots ranged from $12.01^{\circ}$ to $124.2^{\circ}\left(42.36 \pm 24.33^{\circ}\right)$.

Conclusions: Among the root apices of the maxillary molars, the mesiobuccal root apex of the left second molar was closest to the MSF, and it had the highest incidence of protrusion into the sinus. The unique anatomical relationship between the maxillary molars and the MSF in this Chinese population is critical for treatment planning for dental implantation or endodontic procedures.
\end{abstract}

Keywords: Cone beam computed tomography, Maxillary molar, Maxillary sinus

\section{Background}

The anatomical relationship between the root apex of the maxillary molars and the inferior wall of the maxillary sinus (i.e., the maxillary sinus floor, MSF) is critical for planning dental implantation, tooth extraction, and endodontic procedure s[1-4]. The vertical relationship between the MSF and the maxillary root apices varies according to age and

\footnotetext{
* Correspondence: shixiaojun@5thhospital.com; slenll@163.com

${ }^{+}$Xi Zhang and Yan Li contributed equally to this work.

${ }^{1}$ Department of Stomatology, Shanghai Fifth People's Hospital, Fudan

University, Shanghai 200240, China

Full list of author information is available at the end of the article
}

the size and degree of pneumatization of the maxillary sinu $\mathrm{s}[5]$. Occasionally, there is only one layer of mucous or cortical bone in the MSF, and this increases the risk of oroantral fistula or infection in the maxillary sinus. Therefore, identification of the degree of proximity and the thickness of the mucosa and cortical bone between the root apex and the MSF is critical for surgical procedure $s[6,7]$.

Conventional radiographic exams, including periapical and panoramic radiographs, are commonly used for the study of the anatomical relationship between molar root apices and the MS F[8]. However, these two-dimensional images have limitations that may prevent the correct

(c) The Author(s). 2019 Open Access This article is distributed under the terms of the Creative Commons Attribution 4.0 International License (http://creativecommons.org/licenses/by/4.0/), which permits unrestricted use, distribution, and reproduction in any medium, provided you give appropriate credit to the original author(s) and the source, provide a link to the Creative Commons license, and indicate if changes were made. The Creative Commons Public Domain Dedication waiver (http://creativecommons.org/publicdomain/zero/1.0/) applies to the data made available in this article, unless otherwise stated. 
interpretation of the relationship between periapical lesions and the MS F[8,9]. Cone beam computed tomography $(\mathrm{CBCT})$ is a three-dimensional imaging method that has been used for craniofacial radiology. Further, CBCT offers multi-planar views and overcomes the limitations associated with two-dimensional imaging, such as distortion, magnification, and superimpositio $n[3,10]$.

The anatomy of the midface, including the maxillary sinus, differs significantly among the human specie $\mathrm{s}[11]$. Therefore, it is reasonable to postulate that the anatomical proximity between the molar root apex and the MSF varies between populations. Although many studies have focused on the vertical relationship between the maxillary molar root apices and the MSF in Brazilians, Russian, Korean and Turkey,[7, 12-14] detailed anatomical studies of the proximity of molar root apices and the MSF in the Chinese population are rar e[15, 16].

Therefore, this study aimed to evaluate the anatomical relationship between the maxillary molar root apices and the MSF in a Chinese population using CBCT, by measuring the vertical relationships between the MSF and the roots of the molars, distance between the molar root apices and the MSF, thickness of the mucosa and cortical bone of the MSF closest to the root apices, and angle between the buccal and palatal roots.

\section{Methods}

\section{Samples}

This research was approved by the Ethics Committee of Shanghai Fifth People's Hospital, Fudan University (protocol \#2016023) in 2016. Written informed consent to participate was obtained from all of the participants. The CBCT data was collected from 200 patients (96 male and 104 female; mean age, 34 years; range, $18-50$ years) who visited the out-patient clinic of stomatology. The sample consisted of 800 maxillary first and second molars.

Patients were excluded from participation if one maxillary molar was missing from either side of the mouth (excluding the third molars) or if they had a history of orthodontic treatment, root canal therapy for the posterior teeth, significant periodontal disease/bone loss, fused roots or less or more than 3 roots, systematic bone disease, or a tumor involving the maxillary bone.

\section{Image collection and measurements}

CBCT images were obtained with the Planmeca ProMax ${ }^{\circ}$ 3D Max (Planmeca, Finland). The technical data were as follows: anode voltage, $90 \mathrm{kV}$; anode current, $12 \mathrm{~mA}$; and scan time, $27 \mathrm{~s}$. The voxel sizes used for reconstruction were $80 \mu \mathrm{m}$, and the multi-planar views were completed with Romexis software (Planmeca Romexis ${ }^{\circ}$ ) by one oral radiologist. The sections for measuring were selected when the tip of the root was presented in the coronal or sagittal plane.
The measurements included the distance between the root apices of the maxillary molars and the MSF, the thickness of the mucosa and cortical bone of the MSF at the root apices, and the angle between the buccal and palatal roots. The detailed items included the distance between the mesiobuccal root apex and the inferior wall of the MSF (DMBR), the distance between the distobuccal root apex and the inferior wall of the MSF (DDBR), the distance between the palatal root apex and the inferior wall of the MSF (DPR), the cortical thickness of the MSF closest to the mesiobuccal root apex (CTMBR), the cortical thickness of the MSF closest to the distobuccal root apex (CTDBR), the cortical thickness of the MSF closest to the palatal root apex (CTPR), the mucosa thickness of the MSF closest to the mesiobuccal root apex (MTMBR), the mucosa thickness of the MSF closest to the distobuccal root apex (MTDBR), and the mucosa thickness of the MSF closest to the palatal root apex (MTPR) (Fig. 1).

CBCT cross-sectional images were used to evaluate the vertical relationships between the root apices of the maxillary molars and the MSF, and these relationships were classified into five categories (Type 1-5) according to the following criteria described by Kwak et al .[12]: Type I, the MSF is located above the connection between the buccal and palatal root apices; Type II, the MSF is located below the connection between the buccal and palatal root apices, without an apical protrusion over the MSF; Type III, an apical protrusion is observed over the MSF at the buccal root apex; Type IV, an apical protrusion is observed over the MSF at the palatal root apex; and Type V, apical protrusions are observed over the MSF at the buccal and palatal root apices (Fig. 2a-e). The angle between the lines from the upper tangent point of the curvature of furcation to the point of the end tangent point of each root apex was calculated.

\section{Statistical analysis}

Statistical analysis was performed using SPSS 17.0. Data are presented as the mean \pm standard deviation (SD). Unpaired $t$-tests were used to test whether there was any difference between men and women, and paired t-tests were used to test whether there was any difference between left and right sites. For all analyses, $p<0.05$ was considered statistically significant.

\section{Results}

\section{Comparison of sex and location on the relationship between the maxillary molars and the MSF}

We evaluated the influence of sex (male vs female) and location (left vs right side) on the anatomical relationship between the maxillary molars and maxillary sinuses in 104 women and 96 men. Our results demonstrated that the distance between the root apex of the maxillary molars and the MSF, cortical bone thickness of the MSF, 


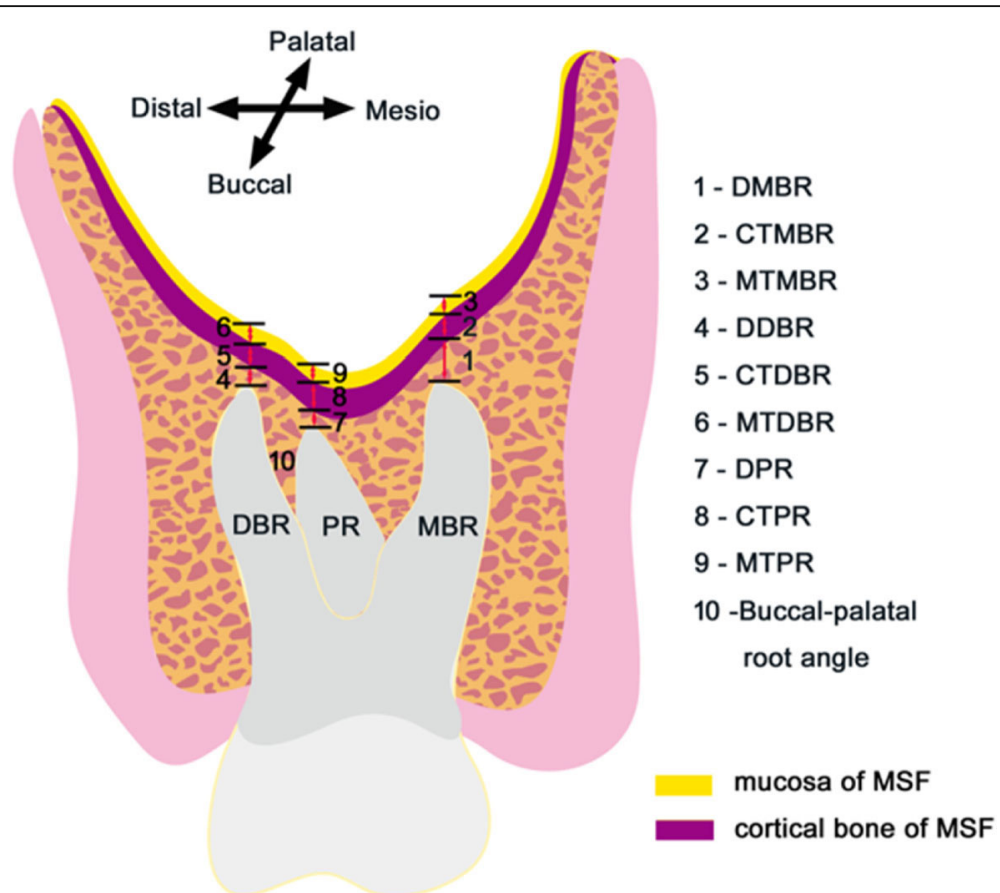

Fig. 1 Measurements of maxillary molars using CBCT cross-sectional images. The measurements include 1-the distance between the mesiobuccal root apex and the inferior wall of the MSF (DMBR), 2-the cortical thickness of the MSF closest to the mesiobuccal root apex (CTMBR), 3-the mucosa thickness of the MSF closest to the mesiobuccal root apex (MTMBR), 4-the distance between the distobuccal root apex and the inferior wall of the MSF (DDBR), 5-the cortical thickness of the MSF closest to the distobuccal root apex (CTDBR), 6-the mucosa thickness of the MSF closest to the distobuccal root apex (MTDBR), 7-the distance between the palatal root apex and the inferior wall of the MSF (DPR), 8-the cortical thickness of the MSF closest to the palatal root apex (CTPR), 9-the mucosa thickness of the MSF closest to the palatal root apex (MTPR), and 10-the angle between the buccal and palatal roots

mucosal thickness of the MSF, maxillary molar root angle, the rates of different types of relationships (Type 1-5) between the maxillary molar roots and the MSF, and the proportion of maxillary sinus penetration were not significantly different between men and women or the left and right sides $(P>0.05)$.

\section{The distance between the root apices of the maxillary molars and the MSF}

We compared the distances between the root apices of the maxillary molars and the MSF. The shortest distances between the root apices and the MSF were $1.57 \pm 3.33 \mathrm{~mm}$ (the mesiobuccal root of the left second molar) and $1.61 \pm$ $3.37 \mathrm{~mm}$ (the mesiobuccal root of the right second molar) (Table 1). Among the maxillary molar roots, protrusion over the MSF most commonly occurred in the mesiobuccal root of the left second molar (frequency, 20.5\%) (Table 2).

The thickness of the cortical bone and mucosa of the MSF closest to the root apices

We evaluated the thickness of the cortical bone and mucosa of the MSF closest to the mesiobuccal, distobuccal,
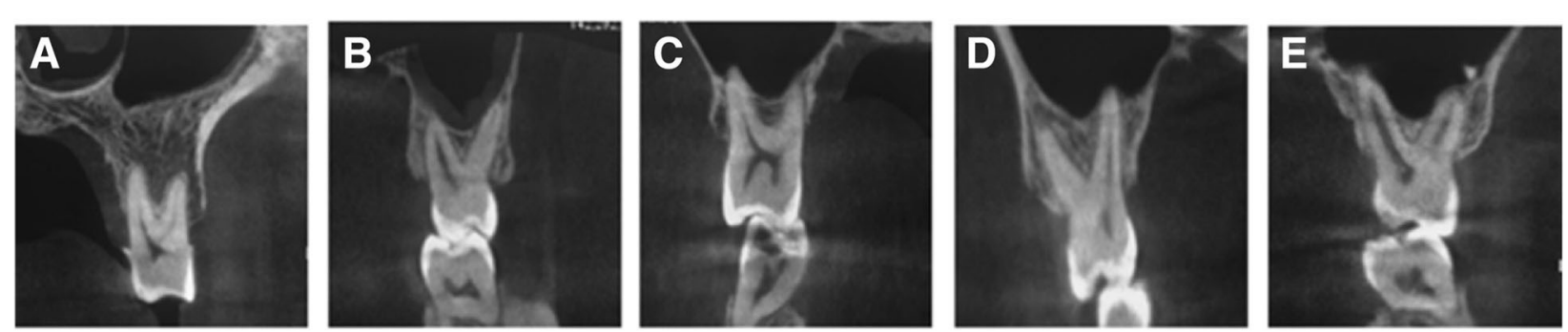

Fig. 2 Different types of vertical relationships between the MSF and the root apices of the maxillary molars. a. Type l: the MSF is located above the connection between the buccal and palatal root apices, $\mathbf{b}$. Type II: the MSF is located below the connection between the buccal and palatal root apices (without an apical protrusion over the MSF), c. Type III: an apical protrusion is observed over the MSF at the buccal root apex, d. Type IV: an apical protrusion is observed over the MSF at the palatal root apex, and $\mathbf{e}$. Type V: apical protrusions are observed over the MSF at the buccal and palatal root apices 
Table 1 Distance between the root apex of the maxillary molars and the MSF

\begin{tabular}{lllll}
\hline tooth position & $\mathrm{n}$ & $\mathrm{MBR}$ & $\mathrm{DBR}$ & $\mathrm{PR}$ \\
\hline 16 & 200 & $2.17 \pm 3.44$ & $2.36 \pm 3.23$ & $2.62 \pm 3.52$ \\
17 & 200 & $1.61 \pm 3.37$ & $1.95 \pm 3.44$ & $2.91 \pm 3.07$ \\
26 & 200 & $2.31 \pm 3.10$ & $2.49 \pm 3.47$ & $2.33 \pm 3.20$ \\
27 & 200 & $1.57 \pm 3.33$ & $1.90 \pm 3.17$ & $2.55 \pm 3.10$
\end{tabular}

Tooth position 16 represents the right first molar, 17 represents the right second molar, 26 represents left first molar, and 27 represents left second molar

$M B R$, the distance between the apex of the mesiobuccal root and the inferior wall of the MSF; $D B R$, the distance between the apex of the distobuccal root and the inferior wall of the MSF; $P R$, the distance between the apex of the palatal root and the inferior wall of the MSF. Unit: $\mathrm{mm}$; data are presented as the mean $S D$

and palatal root apices and compared these thicknesses. Our results revealed that the cortical bone of the MSF was thinnest at the mesiobuccal root apex of the right second molar $(0.45 \pm 0.28 \mathrm{~mm})$ and thickest at the palatal root apex of the left first molar $(0.52 \pm 0.39 \mathrm{~mm})$ (Table 3).

The mucosa of the MSF was thinnest at the distobuccal root of the right second molar $(1.52 \pm 0.85 \mathrm{~mm})$ and thickest at the distobuccal root apex of the left first molar $(1.96 \pm 1.03 \mathrm{~mm})$ (Table 4).

\section{The rates of different types of relationships (type 1-5) between the maxillary molar roots and the MSF}

The rates of the different types of relationships (Type 15) between the maxillary molar roots and the MSF are shown in Table 5. The results demonstrated that Type 1 relationships were most commonly observed in all of the maxillary molars (52.5-62.0\%); Type IV relationships were most uncommonly observed in the right second molar (1.5\%), left first molar (6.0\%), and left second molar (2.0\%); and Type V relationships were most uncommonly observed in the right second molar (3.5\%) (Table 5).

\section{The angle between the buccal and palatal roots}

The angles between the buccal and palatal roots in different molars are shown in Table 6. The smallest angle was observed in the left second molar of Type $\mathrm{V}$ relationships

Table 2 Proportion of apical protrusion of the maxillary molars

\begin{tabular}{lllll}
\hline tooth position & MBR & DBR & PR & Whole tooth \\
\hline 16 & $26(13 \%)$ & $17(8.5 \%)$ & $31(15.5 \%)$ & $47(23.5 \%)$ \\
17 & $32(16 \%)$ & $25(12.5 \%)$ & $10(5 \%)$ & $50(25 \%)$ \\
26 & $16(8 \%)$ & $17(8.5 \%)$ & $27(13.5 \%)$ & $41(20.5 \%)$ \\
27 & $41(20.5 \%)$ & $31(15.5 \%)$ & $14(7 \%)$ & $62(31 \%)$ \\
\hline
\end{tabular}

Tooth position 16 represents the right first molar, 17 represents the right second molar, 26 represents left first molar, and 27 represents left second molar

$M B R$, mesiobuccal root; $D B R$, distobuccal root, $P R$ : the palatal root. Whole tooth, at least one root protruded into the maxillary sinus
Table 3 Cortical thickness of the MSF at the root apices of the maxillary molars

\begin{tabular}{lllll}
\hline tooth position & $\mathrm{n}$ & CTMBR & CTDBR & CTPR \\
\hline 16 & 200 & $0.50 \pm 0.30$ & $0.49 \pm 0.27$ & $0.50 \pm 0.31$ \\
17 & 200 & $0.45 \pm 0.28$ & $0.48 \pm 0.34$ & $0.50 \pm 0.46$ \\
26 & 200 & $0.51 \pm 0.49$ & $0.50 \pm 0.43$ & $0.52 \pm 0.39$ \\
27 & 200 & $0.49 \pm 0.29$ & $0.50 \pm 0.67$ & $0.47 \pm 0.35$
\end{tabular}

Tooth position 16 represents the right first molar, 17 represents the right second molar, 26 represents left first molar, and 27 represents left second molar. CTMBR, cortical thickness of the MSF at the mesiobuccal root apex; CTMDR, cortical thickness of the MSF at the distobuccal root apex; CTPR, cortical thickness of the MSF at the palatal root apex. $n$ teeth number; unit: $\mathrm{mm}$; data are presented as the mean $S D$

$\left(31.55 \pm 26.03^{\circ}\right)$, and the largest angle was observed in the right first molar of Type I relationships $\left(56.47 \pm 29.30^{\circ}\right)$ (Table 6). The angles between the buccal and palatal roots in Type I were significantly larger than that in the other types $(P<0.05$, one-way ANOVA). There were no significant differences among Type II, Type III, Type IV, and Type V $(P>0.05$, one-way ANOVA).

\section{Discussion}

This study aimed to evaluate the anatomical relationship between the first and second maxillary molars and the maxillary sinus and provide a basis for oral clinical treatment.

The maxillary sinus is filled with liquid at birth. During growth and development, the liquid is gradually absorbed and gas begins to fill the sinus cavity. This process is called maxillary sinus gasificatio n[17]. Studies have shown that maxillary sinus gasification is generally completed at the age of 18 year s[5]. In order to avoid the influence of maxillary sinus gasification and guide adult clinical operations, CBCT images of the completed maxillary sinus gasification were analyzed in this study.

The anatomical relationships between the first and second molars (800 teeth) and the maxillary sinus were divided into five types (Type 1-5). Type I relationships were most commonly observed $(57.125 \%)$, and $21.75,11.13,4.5$, and 5.6\% were Type II, Type III, Type IV, and Type V relationships, respectively. We compared the anatomical differences between different ethnicities. Estrela et al .[7] studied

Table 4 The mucosal thickness of the MSF at the root apices of the maxillary molars

\begin{tabular}{lllll}
\hline tooth position & $\mathrm{n}$ & MTMBR & MTDBR & MTPR \\
\hline 16 & 200 & $1.77 \pm 0.86$ & $1.83 \pm 1.03$ & $1.83 \pm 1.17$ \\
17 & 200 & $1.66 \pm 1.00$ & $1.52 \pm 0.85$ & $1.70 \pm 0.65$ \\
26 & 200 & $1.94 \pm 0.98$ & $1.96 \pm 1.03$ & $1.90 \pm 1.12$ \\
27 & 200 & $1.78 \pm 0.72$ & $1.72 \pm 1.05$ & $1.77 \pm 1.19$ \\
\hline
\end{tabular}

Tooth position 16 represents the right first molar, 17 represents the right second molar, 26 represents left first molar, and 27 represents left second molar. MTMBR, mucosal thickness of the MSF at the mesiobuccal root apex; MTDBR, mucosal thickness of the MSF at the distobuccal root apex; MTPR, mucosal thickness of the MSF at the palatal root apex. $n$ teeth number; unit: $\mathrm{mm}$; data are presented as the mean $S D$ 
Table 5 Types of relationships between the maxillary molar roots and the maxillary sinus floor

\begin{tabular}{|c|c|c|c|c|c|c|}
\hline tooth position & $n$ & Type I & Type II & Type III & Type IV & Type V \\
\hline 16 & 200 & $105(52.5 \%)$ & $47(23.5 \%)$ & $18(9 \%)$ & $17(8.5 \%)$ & $13(6.5 \%)$ \\
\hline 17 & 200 & $120(60 \%)$ & $43(21.5 \%)$ & 27 (13.5\%) & $3(1.5 \%)$ & 7 (3.5\%) \\
\hline 26 & 200 & 108 (54\%) & $56(28 \%)$ & $12(6 \%)$ & $12(6 \%)$ & $15(7.5 \%)$ \\
\hline 27 & 200 & $124(62 \%)$ & $28(14 \%)$ & $32(16 \%)$ & $4(2 \%)$ & $10(5 \%)$ \\
\hline sum & 800 & 457 (57.125\%) & 174 (21.75\%) & 89 (11.13\%) & 36 (4.5\%) & 45 (5.6\%) \\
\hline
\end{tabular}

Tooth position 16 represents the right first molar, 17 represents the right second molar, 26 represents left first molar, and 27 represents left second molar

the anatomic relationship between the maxillary molars and the maxillary sinus in Brazil and reported that Type II relationships were most commonly observed (Table 7). These differences indicate that ethnicity may impact the anatomical relationship between the first or second molars and the maxillary sinus. Yurdabakan et al .[14] studied the anatomic relationship between the maxillary third molars and the maxillary sinus in Turkey and found that the most common vertical relationship was that the teeth roots were not contacting with the sinus floor, which is the same as the maxillary first and second molars in our study (Table 7).

Gu et al .[18] also studied the relationship between the maxillary sinus and the maxillary molars in China. But they only divided the relationship between the maxillary posterior teeth and the MSF into three types: Type OS (the root apex extendingbelow/outside the MSF), Type CO (the root apex contacting with the MSF), Type IS (the root apex extendingabove/inside the MSF). And they mostly focused on the impact of age and the absence of adjacent teeth on the relationship. The research from Ananda et al .[19] in China used another way to study the relationship between the maxillary sinus and the first maxillary molar. They divided the anteroposterior relationship into three categories: Type I (anterior to the mesiobuccal root of the maxillary first molar), Type II (posterior to the distobuccal root of the maxillary first molar), Type III (Anterior wall of the maxillary sinus was between the mesiobuccal and distobuccal roots of the maxillary first molar). Different from them our five type division can show more information.

The left second maxillary molar (31\%) had the highest rate of protrusion into the MSF, and the shortest distance $(1.57 \pm 3.33 \mathrm{~mm})$ from the root apex to the MSF (i.e., the distance from the mesiobuccal root to the MSF). Estrela et al .[7] also evaluated the relationship between the MSF and the root apices of the maxillary posterior teeth. Similar to our findings, Estrela et al .[7] reported that the shortest distance from the root apices to the MSF was the distance from the mesiobuccal root of the second molar to the MSF (Table 7).

The study by Lu et al .[20] in China evaluated maxillary sinus mucosal thickening affected by periodontitis of the maxillary premolars/molars and found that the maxillary sinus mucosal thickening increased dramatically as the severity of apical periodontitis increased. Different from them, we measured the thickness of the cortical bone and mucosa of the MSF in order to understand the impact of the relationship between the maxillary molar root and the maxillary sinus on these structures in healthy condition. The cortical thickness of the MSF was $0.45 \pm 0.28 \mathrm{~mm}$ at the mesiobuccal root of the right second molar in the current study. When cortical thickness is decreased, it is more easily damaged by periapical periodontitis, and inflammation may spread to the MS F[21]. Further, thinner cortical bones are less likely to achieve primary stability of implants than thicker cortical bones after maxillary sinus augmentatio $\mathrm{n}[22,23]$. According to our study, the mucosal thickness of the MSF at the root apices of the maxillary molars was around $2 \mathrm{~mm}$, and the mucosa was thinnest at the distobuccal root of right the second molar $(1.52 \pm 0.85$ $\mathrm{mm})$. Ramanauskaite et al .[24] also measured the thickness of the maxillary sinus membrane and found that tooth vitality, residual alveolar bone height, and periodontal bone loss did not influence the thickness of the maxillary sinus membrane. Different from our study, the thickness of the membrane in each apical location was

Table 6 The angle between the buccal and palatal roots of the maxillary molars

\begin{tabular}{lllllll}
\hline tooth position & $\mathrm{n}$ & Type I & Type II & Type III & Type IV & Type V \\
\hline 16 & 200 & $56.47 \pm 29.30$ & $37.71 \pm 16.67$ & $37.73 \pm 22.74$ & $36.40 \pm 19.04$ & $36.26 \pm 20.13$ \\
17 & 200 & $41.84 \pm 25.88$ & $35.58 \pm 17.55$ & $36.77 \pm 17.30$ & $35.98 \pm 7.17$ & $34.60 \pm 21.52$ \\
26 & 200 & $52.88 \pm 28.47$ & $38.89 \pm 28.47$ & $35.68 \pm 17.03$ & $37.29 \pm 19.74$ & $38.33 \pm 19.31$ \\
27 & 200 & $40.99 \pm 24.28$ & $35.69 \pm 17.70$ & $36.09 \pm 14.83$ & $37.56 \pm 11.86$ & $31.55 \pm 26.03$ \\
\hline
\end{tabular}

Tooth position 16 represents the right first molar, 17 represents the right second molar, 26 represents left first molar, and 27 represents left second molar. Unit:; data are presented as the mean $S D$ 


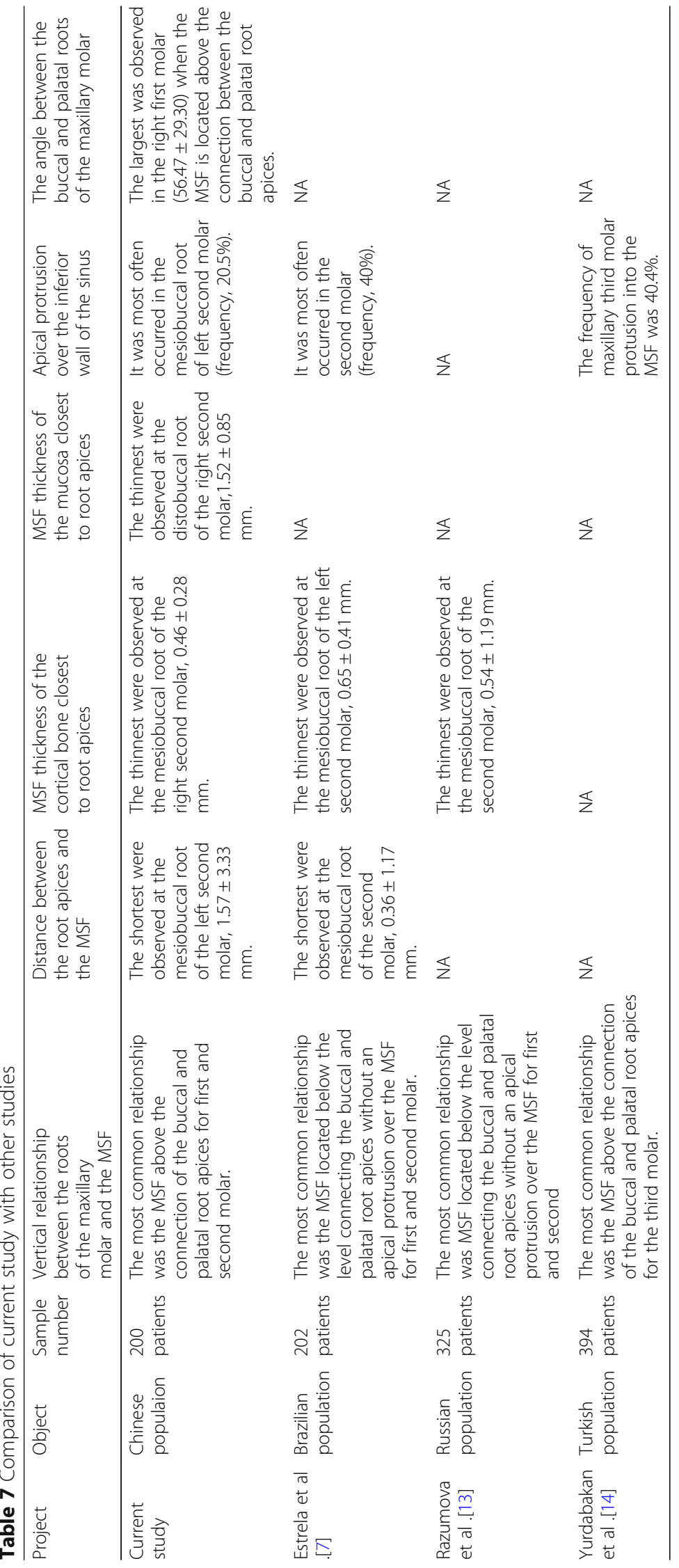


not shown in the study of Ramanauskaite et a 1[24] Here we find that the thickness of membrane in each root apex differs significantly among roots from different people. Therefore, the thickness of membrane should be taken into account when assessing the risk before the treatment.

In the current study, the angle between the buccal and palatal roots is maximum in the right first molar of Type I $(56.47 \pm 29.30)$, and minimum in the left second molar of Type V $(31.55 \pm 26.03)$. Type I had larger angle than the other types (Type II-V). To our knowledge, this finding has not been published before.

Kwak et al .[12] and Tian et al .[16] analyzed the morphological characteristics of the maxillary sinus in Korean and Chinese patients and reported that the shortest distances were observed at the maxillary second molar (Table 7). However, the sample size used in the study conducted by Kwak et al .[12] was only 33, and Tian et al .[16] did not provide a detailed classification of the relationship between the roots and maxillary sinuses of the maxillary molars.

Experimental results have indicated that the anatomical relationship between the maxillary molars and the MSF is complex and impacts clinical operation s[4]. If clinicians do not fully understand the anatomical relationship between the affected teeth and the maxillary sinus through $\mathrm{CBCT}$ imaging, complications, such as maxillary sinus perforation or maxillary sinus infection, may occur during maxillary molar extraction $\mathrm{s}[25]$. CBCT examination can estimate the risk of immediate implant surgery and help reduce the risk of failur e[26]. Understanding the relationship between the root tip and the maxillary sinus mucosa can prevent odontogenic maxillary sinusitis caused by root canal therap y[27].

In this study, the relationship between root apex of each tooth position and MSF definitely help clinicians to predict the risk of maxillary sinus fistula in the treatments of dental implant or root cannel therapy. Due to the significant individual variations, the CBCT scanning is recommended to assess the individual risk. However, based on the concept of ALARA (as low as reasonably achievable), the CBCT scanning only should be necessary when the operation is very close to the MSF.

\section{Conclusions}

Among the root apices of the maxillary molars, the mesiobuccal root apex of the left second molar is closest to the MSF, and it has highest incidence of protrusion into the sinus. The unique anatomic relationships between the maxillary molars and the maxillary sinus in Chinese population allows dentists to provide adequate treatments; estimate the risk of maxillary posterior tooth extractions, dental implantations, and root canals; and reduce the incidence of maxillary sinusitis and other complications.

\section{Supplementary information}

Supplementary information accompanies this paper at https://doi.org/10. 1186/s12903-019-0969-0.

Additional file 1. Raw data of the measurement.

\section{Abbreviations}

CBCT: Cone-Beam Computed Tomography; CTPR: The cortical thickness of the MSF closest to the palatal root apexi; DDBR: The distance between the distobuccal root apex and the inferior wall of the MSF; DMBR: The distance between the mesiobuccal root apex and the inferior wall of the MSF; DPR: The distance between the palatal root apex and the inferior wall of the MSF CTMBRThe cortical thickness of the MSF closest to the mesiobuccal root apex CTDBRThe cortical thickness of the MSF closest to the distobuccal root apex; MSF: Maxillary sinus floor; MTMBR: The mucosa thickness of the MSF closest to the mesiobuccal root apex MTDBRThe mucosa thickness of the MSF closest to the distobuccal root apex; MTPR: The mucosa thickness of the MSF closest to the palatal root apex

\section{Acknowledgements}

This work was supported by Shanghai Municipal Planning Commission of Science and Research Fund (no. 201740230), Talent Development Plan funded by Shanghai Fifth People's Hospital, Fudan University (no. 2017WYRCJY02), Minhang District Planning Commission of Science and Research Fund (no. 2019MHZ039).

\section{Authors' contributions}

LS and XS developed the concept and design of this study. XZ and YL performed the clinical examination and contributed to the analysis of behavioral data. LS supervised the data assessment. $X Z, Y L$, and BX performed statistical analyses, and all authors (XZ, YL, YZ, FH, BX, XS, and LS) contributed to the interpretation of the data. $X Z, Y L$, and $L S$ drafted the manuscript. All authors $(X Z, Y L, Y Z, F H, B X, X S$, and $L S)$ read and approved the final manuscript.

\section{Funding}

This work was supported by Shanghai Municipal Planning Commission of Science and Research Fund (no. 201740230), Talent Development Plan funded by Shanghai Fifth People's Hospital, Fudan University (no.

2017WYRCJY02), Minhang District Planning Commission of Science and Research Fund (no. 2019MHZ039). The funding bodies played no role in the design of the study and collection, analysis, and interpretation of data and in writing the manuscript.

\section{Availability of data and materials}

The raw data can be found in Additional file 1: raw data to BMC oral health.

Ethics approval and consent to participate

This research was approved by the Ethics Committee of Shanghai Fifth

People's Hospital, Fudan University (protocol \#2016023).

Consent for publication

Not applicable.

Competing interests

There is no financial or non-financial competing interests.

\section{Author details}

${ }^{1}$ Department of Stomatology, Shanghai Fifth People's Hospital, Fudan University, Shanghai 200240, China. 'Department of Health Management, The Third Xiangya Hospital, Central South University, Changsha 410013, China. ${ }^{3}$ Radiology Department, Shanghai Prison General Hospital, Shanghai 201318, China.

Received: 20 July 2019 Accepted: 22 November 2019 Published online: 16 December 2019

\section{References}

1. Silva MA, Wolf $U$, Heinicke F, Bumann A, Visser H, Hirsch E. Cone-beam computed tomography for routine orthodontic treatment planning: a radiation dose evaluation. Am J Orthod Dentofacial Orthop. 2008;133(5): 640 e641-5. 
2. Estrela $C$, Holland R, Estrela CR, Alencar AH, Sousa-Neto MD, Pecora JD. Characterization of successful root canal treatment. Braz Dent J. 2014; 25(1):3-11.

3. Weiss R 2nd, Read-Fuller A. Cone Beam Computed Tomography in Oral and Maxillofacial Surgery: An Evidence-Based Review. Dent J (Basel). 2019;7(52):1-23.

4. Lavasani SA, Tyler C, Roach SH, McClanahan SB, Ahmad M, Bowles WR. Cone-beam computed tomography: anatomic analysis of maxillary posterior teeth-impact on endodontic microsurgery. J Endod. 2016;42(6):890-5.

5. Lorkiewicz-Muszynska D, Kociemba W, Rewekant A, Sroka A, JonczykPotoczna K, Patelska-Banaszewska M, Przystanska A. Development of the maxillary sinus from birth to age 18. Postnatal growth pattern. Int J Pediatr Otorhinolaryngol. 2015;79(9):1393-400.

6. Kang SH, Kim BS, Kim Y. Proximity of posterior teeth to the maxillary sinus and Buccal bone thickness: a biometric assessment using cone-beam computed tomography. J Endod. 2015:41(11):1839-46.

7. Estrela C, Nunes CA, Guedes OA, Alencar AH, Estrela CR, Silva RG, Pecora JD, Sousa-Neto MD. Study of anatomical relationship between posterior teeth and maxillary sinus floor in a subpopulation of the Brazilian central region using cone-beam computed tomography - part 2. Braz Dent J. 2016;27(1):9-15.

8. Lopes LJ, Gamba TO, Bertinato JV, Freitas DQ. Comparison of panoramic radiography and CBCT to identify maxillary posterior roots invading the maxillary sinus. Dentomaxillofac Radiol. 2016;45(6):20160043.

9. Hassan BA. Reliability of periapical radiographs and orthopantomograms in detection of tooth root protrusion in the maxillary sinus: correlation results with cone beam computed tomography. J Oral Maxillofac Res. 2010;1(1):e6.

10. Horner K, O'Malley L, Taylor K, Glenny AM. Guidelines for clinical use of CBCT: a review. Dentomaxillofac Radiol. 2015;44(1):20140225.

11. Lacruz RS, Stringer CB, Kimbel WH, Wood B, Harvati K, O'Higgins P, Bromage TG, Arsuaga JL. The evolutionary history of the human face. Nat Ecol Evol. 2019;3(5):726-36.

12. Kwak HH, Park HD, Yoon HR, Kang MK, Koh KS, Kim HJ. Topographic anatomy of the inferior wall of the maxillary sinus in Koreans. Int J Oral Maxillofac Surg. 2004;33(4):382-8.

13. Razumova S, Brago A, Howijieh A, Manvelyan A, Barakat H, Baykulova M. Evaluation of the relationship between the maxillary sinus floor and the root apices of the maxillary posterior teeth using cone-beam computed tomographic scanning. J Conserv Dent. 2019;22(2):139-43.

14. Yurdabakan ZZ, Okumus O, Pekiner FN. Evaluation of the maxillary third molars and maxillary sinus using cone-beam computed tomography. Niger J Clin Pract. 2018:21(8):1050-8.

15. Wei MY, Wang XL, Li Y. Gao Y: [study on root canal variation of maxillary premolar and its relationship to maxillary sinus by cone-beam $C T$. Shanghai Kou Qiang Yi Xue. 2018;27(2):156-63.

16. Tian XM, Qian L, Xin XZ, Wei B, Gong Y. An analysis of the proximity of maxillary posterior teeth to the maxillary sinus using cone-beam computed tomography. J Endod. 2016;42(3):371-7.

17. Aust R, Stierna P, Drettner B. Basic experimental studies of ostial patency and local metabolic environment of the maxillary sinus. Acta Otolaryngol Suppl. 1994;515:7-10 discussion 11.

18. Gu Y, Sun C, Wu D, Zhu Q, Leng D, Zhou Y. Evaluation of the relationship between maxillary posterior teeth and the maxillary sinus floor using conebeam computed tomography. BMC Oral Health. 2018;18(1):164.

19. Ananda GK, Nambiar P, Mutalik S, Shanmuhasuntharam P. Anatomical considerations for implant placements in first maxillary molar extracted sites in east Asian patients. Surg Radiol Anat. 2015;37(9):1099-108.

20. Lu Y, Liu Z, Zhang L, Zhou X, Zheng Q, Duan X, Zheng G, Wang H, Huang D. Associations between maxillary sinus mucosal thickening and apical periodontitis using cone-beam computed tomography scanning: a retrospective study. J Endod. 2012;38(8):1069-74.

21. Chand A, Ronghe B, Byakod G, Lathi AA, Muglikar S. Relationship between inferior wall of maxillary sinus and maxillary posterior teeth using conebeam computed tomography in healthy and chronic periodontitis patients. J Indian Soc Periodontol. 2017;21(6):466-72.

22. Moriwaki H, Yamaguchi S, Nakano T, Yamanishi Y, Imazato S, Yatani H. Influence of implant length and diameter, Bicortical Anchorage, and sinus augmentation on bone stress distribution: three-dimensional finite element analysis. Int J Oral Maxillofac Implants. 2016;31(4):e84-91.

23. Salem D, Alshihri A, Arguello E, Jung RE, Mohmed HA, Friedland B. Volumetric analysis of Allogenic and Xenogenic bone substitutes used in maxillary sinus augmentations utilizing cone beam $\mathrm{CT}$ : a prospective randomized pilot study. Int J Oral Maxillofac Implants. 2019;34(4):920-6.
24. Ramanauskaite A, Ataman-Duruel ET, Duruel O, Tozum MD, Yildirim T, Tozum TF. Effects of clinical local factors on thickness and morphology of Schneiderian membrane: a retrospective clinical study. Clin Implant Dent Relat Res. 2019;21(4):715-22.

25. Hirata Y, Kino K, Nagaoka S, Miyamoto R, Yoshimasu H, Amagasa T. a clinical investigation of oro-maxillary sinus-perforation due to tooth extraction. Kokubyo Gakkai Zasshi. 2001;68(3):249-53.

26. Liu H, Liu R, Wang M, Yang J. Immediate implant placement combined with maxillary sinus floor elevation utilizing the transalveolar approach and nonsubmerged healing for failing teeth in the maxillary molar area: a randomized controlled trial clinical study with one-year follow-up. Clin Implant Dent Relat Res. 2019;21(3):462-72.

27. Estrela C, Porto OC, Costa NL, Garrote Mda S, Decurcio DA, Bueno MR, Silva BS. Large Reactional Osteogenesis in maxillary sinus associated with secondary root canal infection detected using cone-beam computed tomography. J Endod. 2015;41(12):2068-78.

\section{Publisher's Note}

Springer Nature remains neutral with regard to jurisdictional claims in published maps and institutional affiliations.
Ready to submit your research? Choose BMC and benefit from:

- fast, convenient online submission

- thorough peer review by experienced researchers in your field

- rapid publication on acceptance

- support for research data, including large and complex data types

- gold Open Access which fosters wider collaboration and increased citations

- maximum visibility for your research: over $100 \mathrm{M}$ website views per year

At $\mathrm{BMC}$, research is always in progress.

Learn more biomedcentral.com/submissions 